\title{
Progress in the treatment of solid tumors with apatinib: a systematic review
}

This article was published in the following Dove Press journal:

OncoTargets and Therapy

\section{Deze Zhao \\ Helei Hou \\ Xiaochun Zhang}

Department of Medical Oncology, The Affiliated Hospital of Qingdao University, Qingdao University,

Qingdao 266003, China
Correspondence: Xiaochun Zhang Department of Medical Oncology, The Affiliated Hospital of Qingdao University, Qingdao University, 16 Jiangsu Road, Qingdao 266003, China Email zhangxiaochun9670@I26.com

\begin{abstract}
With the investigation of molecular targets, many agents, such as trastuzumab and ramucirumab, have attained a positive outcome in oncotherapy. Vascular endothelial growth factor (VEGF) is considered a potent factor in angiogenesis and plays an important role in the growth of tumors. Moreover, both VEGF and its receptor are usually excessively expressed in solid tumors and could be hopeful targets for the treatment of neoplasms. Apatinib (YN968D1) is an oral small-molecule tyrosine kinase inhibitor of VEGFR-2. By inhibiting several signaling transduction pathways, it restrains angiogenesis and subsequently controls tumorigenesis. According to current studies, apatinib shows promising application in various solid tumors as a post-second- and post-third-line treatment. It could significantly improve the median overall survival and progression-free survival of patients with tolerated adverse reactions. This paper aims to summarize the recent research on apatinib including the mechanism, pharmacokinetics, trials, adverse reactions, and prospect as a treatment.
\end{abstract}

Keywords: VEGF, VEGFR-2, apatinib, angiogenesis, solid tumors, gastric cancer

\section{Introduction}

Solid tumors, as the primary cause of cancer death, have caused great societal and economic effects. ${ }^{1}$ Generally, surgery is the most effective treatment for early-stage tumors, but most patients experience recurrence after radical surgery. ${ }^{2}$ In recent years, the development and application of targeted drugs have enabled cancer treatment to enter the "precise" era. ${ }^{3}$ In addition, the combined use of targeted therapy, immunotherapy, and traditional chemotherapy has innovated the standard treatment for many malignancies, bringing new light to patients with recurrent tumors. ${ }^{4}$

A tumor requires large amounts of oxygen and nutrients for maintaining its activity, which causes large quantities of blood vessels to develop, creating a vicious cycle. ${ }^{5}$ Therefore, pathological angiogenesis is considered a hallmark of tumorigenesis, and tumor development can be curbed by blocking this process. ${ }^{6}$ Vascular endothelial growth factor (VEGF) signaling is the primary rate-limiting step in angiogenesis, which is extremely active in most tumors. ${ }^{8,9}$ Angiogenesis is mainly mediated by two receptor tyrosine kinases VEGFR-1 and VEGFR-2. ${ }^{10}$ VEGFR-1 has a high affinity for VEGF binding, but the kinases activated by VEGFR-2 appear more energetic and contribute more to angiogenesis. ${ }^{10,11}$ In addition, VEGFR-1 is expressed on vascular endothelial cells as well as many other cells, such as hematopoietic stem cells, monocytes, and macrophages, but the expression of VEGFR-2 is restricted to endothelial cells. ${ }^{8,12}$ Therefore, VEGFR-2 is a promising antitumor target.

Apatinib mesylate is a new small-molecule tyrosine kinase inhibitor (TKI) of VEGFR-2. It can significantly inhibit the angiogenesis of neoplasms and has 
demonstrated tolerance, safety, and efficacy in the clinic. ${ }^{13}$ To date, apatinib has been investigated in Phase I/II/III trials and has shown positive results in multiple tumors, such as gastric cancer (GC), breast cancer, lung cancer, and esophageal cancer. ${ }^{14-21}$

\section{The therapeutic mechanism of apatinib}

During tumor formation, environmental changes such as hypoxia and genetic mutations to molecules such as $K$-ras, p53, or $H E R 2 / E r b B 2$ induce the expression of VEGF in cancer cells. ${ }^{22}$ Meanwhile, VEGFR-2 expression is also upregulated in tumor vasculature compared to the normal vascular system. ${ }^{12,23}$ After the binding of VEGF and VEGFR-2, the carboxy-terminal TK domain of the receptor undergoes autophosphorylation, leading to downstream signaling transduction. Apatinib can block the phosphorylation of VEGFR-2 by binding its intracellular adenosine triphosphate sites, thereby restraining several signaling pathways: the Raf/MEK/Erk pathway, which results in the proliferation of endothelial cells; the p38-MAPK pathway, which stimulates the migration of endothelial cells; and the $\mathrm{PI} 3 \mathrm{~K} / \mathrm{AKT} / \mathrm{mTOR}$ pathway, which strengthens the survival of endothelial cells and enhances vascular permeability
(Figure 1). ${ }^{12,24}$ Additionally, the kinase activities of Ret, c-kit, and c-src have been proven to be suppressed by apatinib in vitro in enzyme experiments, but the specific mechanisms need further exploration. ${ }^{25,26}$ As a result, apatinib can effectively restrain proliferation, migration, and permeability of vascular endothelial cells.

Recently, many studies have shown that apatinib could directly act on tumor cells and promote their apoptosis. Apatinib mediates this process by inhibiting the PI3K/Akt signaling pathway and subsequently upregulating the expression of proapoptotic genes Bax and caspase- 9 and downregulating the expression of the anti-apoptotic gene $B c l-2$. Further related mechanism research is in progress. ${ }^{27-31}$ These studies may broaden the therapeutic indications for drugs and provide guidance for drug regimens.

In addition, multidrug resistance (MDR) of tumor cells is an intractable obstacle for cancer treatment. ${ }^{32}$ The mechanism of MDR is sophisticated, and a study has shown that it can be ascribed to the high expression of cell membrane-bound adenosine triphosphate-binding cassette (ABC) transporters. ${ }^{33}$ After the signal activates the receptor, the transporter extrudes various types of chemotherapeutic agents out of tumor cells, hence reducing the cytotoxic effect. ${ }^{33}$ Not by blocking the ERK1/2 or AKT pathway or

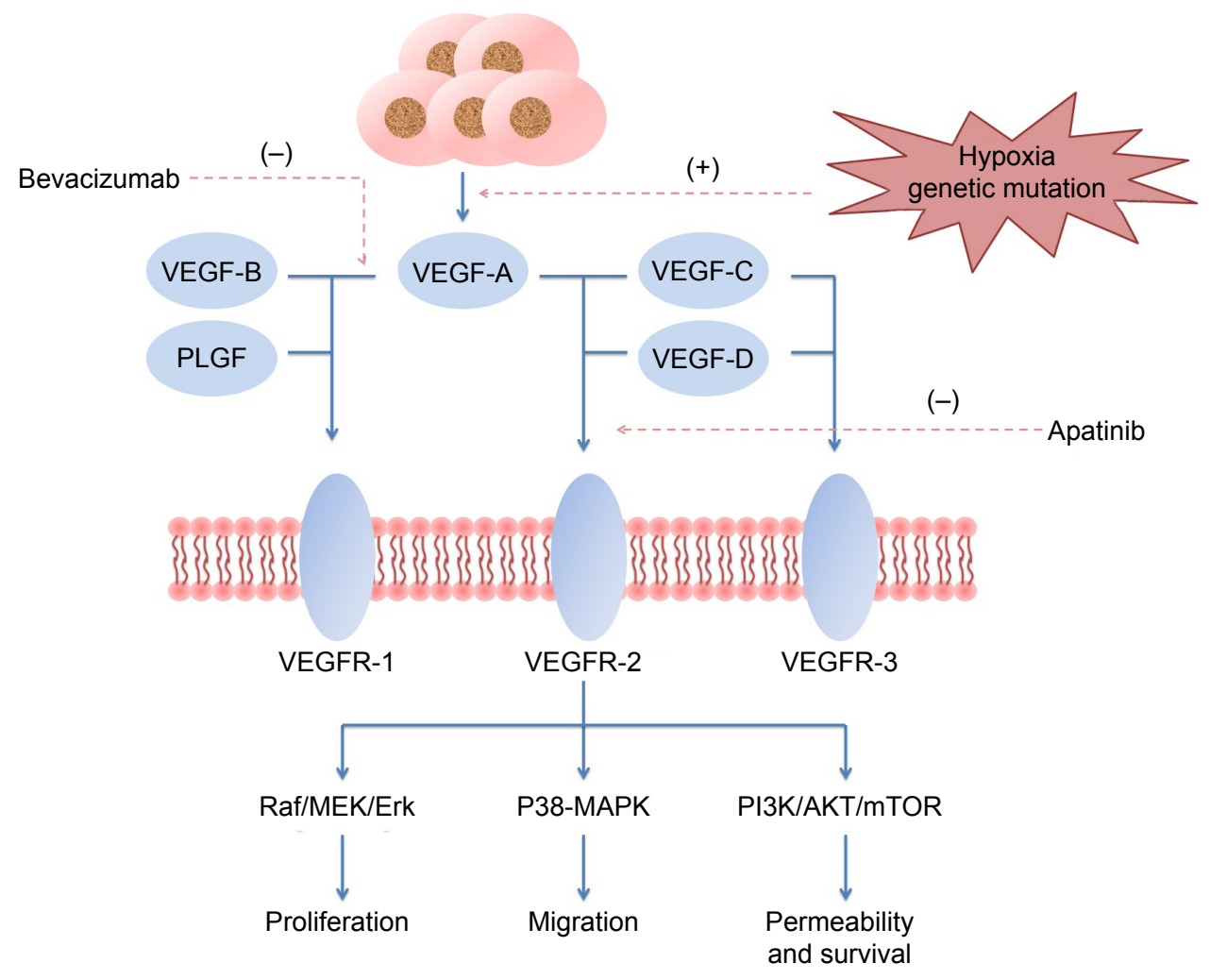

Figure I The therapeutic mechanism of apatinib. ${ }^{12,22-24}$

Abbreviations: PLGF, placental growth factor; VEGF, vascular endothelial growth factor. 
decreasing the expression of $\mathrm{ABCB} 1$ or $\mathrm{ABCG} 2$, apatinib could act directly on $\mathrm{ABCB} 1$ or $\mathrm{ABCG} 2$ and inhibit its efflux function. Thus, apatinib reverses the MDR caused by ABC proteins. ${ }^{34}$ This discovery proved that apatinib combined with traditional chemotherapeutic drugs could achieve effective results and resist the emergence of MDR.

\section{Pharmacokinetic study of apatinib}

Apatinib is mainly metabolized by cytochrome P450 (CYP)3A4/5 and CYP2D6, and the major routes of apatinib bioconversion contain $E$ - and $Z$-cyclopentyl-3-hydroxylation, $N$-dealkylation, pyridyl-25- $N$-oxidation, 16-hydroxylation, dioxygenation, and $O$-glucuronidation after 3 -hydroxylation. Nine primary metabolites have been verified by comparing them with the reference standards (M1-1, M1-2, M1-6, M4-7, M4-8, M6-9, M6-10, M8-2, and M9-2). Patients reach the $\mathrm{C}_{\max }$ of apatinib 2.9-4.7 hours after taking the medication. The cumulative recovery of the administered dose is $76.8 \%$, which is mainly excreted in the feces $(69.8 \%$ of the dose). Only a small proportion is eliminated via urine (7.02\% of the dose). Moreover, $\sim 59.0 \%$ of the intake dose is egested unaltered via feces and inappreciable amounts of unchanged apatinib are found in urine, which indicates that apatinib is widely metabolized in the biosystem. ${ }^{35}$ These data could be used to predict potential drug interactions and pharmacokinetic variability and provide guidance for later experimental design.

A population pharmacokinetic model for apatinib was established and analyzed using data from 106 individuals, including healthy volunteers and patients with malignant tumors. The ultimate model was a two-compartment model that was composed of a complex absorption model including mixed first- and zero-order processes and firstorder elimination. The absolute clearance (CL/F) of apatinib was $57.8 \mathrm{~L} / \mathrm{h}$, and its apparent volume at steady state was 112.5 L. Three factors influenced the pharmacokinetics of apatinib: dose, cancer status, and gastric surgery state. The exposure of apatinib was proportionately less than the dose increase, and the dose with a reduction in bioavailability was $766 \mathrm{mg}$. In addition, the administered dose of $425 \mathrm{mg}$ twice daily appeared to have a higher exposure than the dose of $850 \mathrm{mg}$ once daily. ${ }^{36}$ This study provided a detailed description of the factors that influenced the metabolism of apatinib in the human body, providing guidance for subsequent medications.

A research study was implemented to assess the drug interactions of apatinib with a CYP3A4 inducer (rifampin) or a CYP3A inhibitor (itraconazole). In comparison to the single administration of apatinib, the combination of apatinib and rifampin led to a 5.6-fold absolute clearance (CL/F) and an $83 \%$ reduction in plasma area under the curve $\mathrm{AUC}_{0-\mathrm{t}}$ of apatinib. On the contrary, the combination of apatinib and itraconazole decreased the absolute clearance by $40 \%$ and enhanced its $\mathrm{AUC}_{0-\mathrm{t}}$ by $75 \% .{ }^{37}$ It proved that coadministration of apatinib with a CYP3A4 inducer had a strong influence on the pharmacokinetics of apatinib, while the CYP3A inhibitor had a weak influence. This suggests that we need to pay attention to the interaction between drugs in the formulation of future treatment plans.

\section{The clinical research regarding apatinib Gastric cancer}

At present, the number of newly diagnosed GC patients has increased by $\sim 679,100$ each year, and GC has become the second leading primary cause of cancer death in China. Because of the low diagnosis rates, almost $80 \%$ of patients were found at advanced cancer stages. ${ }^{38}$ However, even after receiving first- and second-line cytotoxic chemotherapy, many of them are not cured or relapse a few years later. At present, there are no treatment criteria for patients who have no response to second-line chemotherapy. ${ }^{39}$

In 2010, a Phase I study was conducted on apatinib, which determined the maximum tolerated dose to be $850 \mathrm{mg}$ once daily. The dose-limiting toxicities were hypertension and hand-foot syndrome that occurred with administration of $1,000 \mathrm{mg}$. Pharmacokinetic analysis revealed apatinib had an early absorption with a half-life of 9 hours. A total of 37 patients were eligible to evaluate tumor response. Seven patients $(18.9 \%)$ had partial response (PR) and 24 patients (64.9\%) achieved stable disease (SD) with a disease control rate of $83.8 \%$ at 8 weeks. ${ }^{40}$ In 2013, a subsequent Phase II study was performed enrolling 144 patients. The result demonstrated that the progression-free survival (PFS) of patients who received apatinib $850 \mathrm{mg}$ once daily was 3.67 months and the PFS of patients who took $425 \mathrm{mg}$ twice daily was 3.20 months, while the PFS of patients who received placebo was 1.40 months; moreover, the median overall survival (OS) was $4.83,4.27$, and 2.50 months, respectively. ${ }^{41}$ These two experiments initially evaluated the efficacy and safety of apatinib in GC patients and provided the basis for the design of clinical trials and the determination of dosing schedules.

In 2016, a randomized, double-blind, placebo-controlled Phase III study for GC was carried out to assess the effectiveness and safety of apatinib. A total of 267 patients from 32 centers in China were assigned to take apatinib $850 \mathrm{mg}$ or 
placebo once daily. The median OS of the apatinib group was 6.5 months (95\% CI: 4.8-7.6), in comparison with 4.7 months (95\% CI: 3.6-5.4) for the placebo group ( $P=0.0149$; hazard ratio: $0.709,95 \% \mathrm{CI}$ : $0.537-0.937 ; P=0.0156)$. In addition, apatinib meaningfully prolonged the median PFS compared with placebo (2.6 vs 1.8 months; hazard ratio: 0.444 , 95\% CI: $0.33-0.61 ; P<0.0001) .{ }^{18}$ Furthermore, the efficacy of apatinib on OS was by prolonging PFS, rather than postprogression survival. ${ }^{42}$ This experiment further proves that apatinib has a significant therapeutic effect on GC patients and has high safety, so it can be used as a drug for treating advanced GC patients.

The effectiveness of many antiangiogenic drugs for advanced GC is listed in Table 1. As we can see, all of them significantly improved the PFS and OS of patients. In terms of therapeutic effects (prolonged PFS and OS), multidrug combination therapy achieved better results. But compared to the control group, the effects of these programs (including ramucirumab and bevacizumab) were unsatisfactory. Among monotherapies, the average median PFS and OS were 2.4 and 6.1 months, respectively, and apatinib provided an above-average effect. As for disease control rate, apatinib, compared to its control group, was the most obvious group that could significantly control the disease. Recently, a network meta-analysis evaluated the efficacy and safety of targeted drugs (mainly including apatinib, regorafenib, rilotumumab, everolimus, bevacizumab, and sunitinib) with or without chemotherapy in advanced GC treatment and demonstrated that apatinib showed the best improvement in PFS, OS, and overall response rate. ${ }^{43}$

To find more detailed clinical effectiveness and safety of apatinib in GC, a real-world study is currently ongoing (ClinicalTrial.gov identifier NCT03333967). In addition, a series of combination drug therapies are being researched to determine the valid therapeutic method. Studies with apatinib in combination with S-1 (ClinicalTrial.gov identifier NCT03271073), XELOX (ClinicalTrial.gov identifier NCT03229096), Paclitaxel (ClinicalTrial.gov identifier NCT03144843), and SOX (ClinicalTrial.gov identifier NCT03007446) are presently underway.

\section{Breast cancer}

Breast cancer is heterogeneous in the clinic. There are three biomarkers, the expression of HER-2 and the receptors for progesterone and estrogen, which are usually used to assist in the choice of therapy. However, triple-negative breast cancer (TNBC) lacks the admitted targets for treatment. So far, several potential actionable pathways have been found in TNBC, such as the RAS/RAF/MEK pathway and the PI3K/ mTOR pathway. ${ }^{44}$ Based on the mechanism, apatinib could be applied in TNBC.

In 2014, a prospective, open-label, Phase II, multicenter trial was implemented to evaluate the effect and safety of apatinib monotherapy in patients with metastatic TNBC. ${ }^{19}$ First, 25 patients were enrolled, who received apatinib $750 \mathrm{mg} /$ day in a 4-week cycle. Then, according to toxicity response, he dose of apatinib was then adjusted to $500 \mathrm{mg} /$ day. Finally, among the 56 appraisable patients, the overall response rate was $10.7 \%$ and the clinical benefit rate was $25.0 \%$. The median OS and PFS were 10.6 (95\% CI: 5.6-15.7) and 3.3 (95\% CI: 1.7-5.0) months, respectively. As a result, the study recommended that a dose of $500 \mathrm{mg}$ /day was suitable for metastatic TNBC. ${ }^{19}$ In 2017, a non-clinical trial evaluated the potency of apatinib in metastatic breast cancer (MBC), and the result was consistent with previous reports. Moreover, it was found that preceding therapy with bevacizumab had no effect on the efficacy of apatinib. ${ }^{45}$

So far, many antiangiogenic agents have shown a disappointing effect in MBC. Sunitinib and sorafenib, VEGF receptor tyrosine kinase inhibitors, were the first batch of

Table I Efficacy of different antiangiogenic drugs in advanced gastric cancer

\begin{tabular}{|c|c|c|c|c|c|c|c|}
\hline Drugs & $\mathbf{n}$ & $\begin{array}{l}\text { Median PFS } \\
\text { (months) }\end{array}$ & $\begin{array}{l}\text { Median OS } \\
\text { (months) }\end{array}$ & ORR (\%) & SD (\%) & DCR (\%) & Reference \\
\hline Sunitinib & 78 & 2.3 & 6.8 & 2.6 & 32.1 & 34.7 & 77 \\
\hline Regorafenib vs placebo & 147 & 2.6 vs 0.9 & 5.8 vs 4.5 & 1 & 1 & 1 & 78 \\
\hline Ramucirumab vs placebo & 355 & 2.1 vs 1.3 & 5.2 vs 3.8 & 3.4 vs 2.6 & 45.4 vs 20.5 & 48.8 vs 23.1 & 60 \\
\hline Apatinib vs placebo & 267 & 2.6 vs 1.8 & 6.5 vs 4.7 & 2.8 vs 0 & 42.1 vs 8.8 & 44.9 vs 8.8 & 18 \\
\hline Sorafenib + oxaliplatin & 40 & 3 & 6.5 & 2.8 & 47.2 & 50.0 & 79 \\
\hline Bevacizumab + chemotherapy vs placebo + chemotherapy & 517 & 6.7 vs 5.3 & 12.1 vs 10.1 & 46.0 vs 37.4 & 29.9 vs 30.3 & 75.9 vs 67.7 & 80 \\
\hline Ramucirumab + paclitaxel vs placebo + paclitaxel & 665 & 4.4 vs 2.9 & 9.6 vs 7.4 & 27.9 vs 16.1 & 52.1 vs 47.5 & 80.0 vs 63.6 & 81 \\
\hline Ramucirumab + FOLFOX vs placebo + FOLFOX & 168 & 6.4 vs 6.7 & $11.7 \mathrm{vs} 11.5$ & 45.2 vs 46.5 & 39.3 vs 20.2 & 84.5 vs 66.7 & 82 \\
\hline
\end{tabular}

Notes: "I" not available. Because there are few targeted drugs for gastric cancer, this summary chooses Phase II or III trials of antiangiogenic drugs with or without chemotherapy in advanced gastric cancer. In terms of comparison, the standard is based on their ability to prolong PFS, OS, or control disease compared to its own control group. Abbreviations: DCR, disease control rate; ORR, overall response rate; OS, overall survival; PFS, progression-free survival; SD, stable disease. 
drugs approved for clinical use. A study reported that high doses of these receptor tyrosine kinase inhibitors could lead to metastasis in a mouse model of breast cancer; ${ }^{46}$ this finding may affect the clinical application of VEGF drugs. To elucidate their mechanism, many researchers conducted experiments and found that sunitinib and sorafenib were multi-target agents, and that the acceleration process may be ascribed to off-target effects. In addition to acting on VEGFR, these drugs also acted on platelet-derived growth factor receptor and KIT, which are important mediums for the recruitment of pericytes. ${ }^{47,48}$ As a result, pericytes decreased and led to hypoxia-associated epithelial-to-mesenchymal transition and metastasis mediated by the Met signaling pathway. ${ }^{49}$ However, apatinib has less activity toward receptors such as platelet-derived growth factor receptor and KIT. ${ }^{25}$ Therefore, compared with other agents, this could be an advantage for the treatment of MBC. Currently, a prospective, multicenter, observational study of apatinib, single or combined with capecitabine, has been conducted in patients with metastatic Her-2-negative breast cancer (ClinicalTrial.gov identifier NCT03086785). This study is designed to provide further data about the effect and toxicity of apatinib. Furthermore, patients with TNBC are currently being recruited to demonstrate the antitumor activity of the combination of apatinib and paclitaxel (ClinicalTrial.gov identifier NCT03348098). A Phase II study of apatinib combined with oral vinorelbine in metastatic Her-2-negative breast cancer is also being conducted (ClinicalTrial.gov identifier NCT02768415).

\section{Lung cancer}

Lung cancer is the predominant cancer type in China, ${ }^{1}$ and non-small-cell lung cancer (NSCLC) is the main histological subtype of the disease. With the increasing cognition of the molecular changes that regulate tumor progression, the management of NSCLC has totally changed. However, frequent resistance to therapy makes advanced NSCLC hard to cure..$^{50}$

In 2017, apatinib was tested as a salvage therapy for patients with advanced NSCLC. Among 42 patients administered a daily dose of $500 \mathrm{mg}, 4$ patients had PR and 22 patients experienced SD with a disease response rate of 9.5\% and a control rate of $61.9 \%$; the median PFS and OS were 4.2 and 6.0 months, respectively. ${ }^{20}$ The study suggested that apatinib could be used as a valid regimen for advanced NSCLC.

In NSCLC, there were $\sim 45 \%$ patients without epidermal growth factor receptor (EGFR) mutations, and these patients were often found to have progressive disease 4-6 months after first-line treatment. ${ }^{51}$ Therefore, to find a suitable second-line treatment, the trials on the treatment of EGFR wild-type lung cancer were launched. ${ }^{52,53}$ A group of 16 patients were enrolled and administered apatinib 250-500 mg/day. Three patients exhibited a PR, and eight patients showed SD. The response rate and control rate were $18.8 \%$ and $68.8 \%$, respectively. The median PFS was 4.4 months. ${ }^{52}$ Thus, apatinib may be a choice for EGFR wildtype patients as a second-line treatment. Presently, the wide use of EGFR-TKIs in NSCLC caused excessive EGFR-TKI resistance. Therefore, methods to combat resistance have become a study hotspot. Recently, it was confirmed that combination therapy with apatinib and other EGFR-TKIs, such as erlotinib and gefitinib, could combat acquired EGFR-TKI resistance in NSCLC. ${ }^{54,55}$ Therefore, apatinib is a good choice as post-second-line treatment for advanced lung cancer.

At present, the Chinese Academy of Medical Sciences is recruiting patients with small cell lung cancer for a Phase II study of apatinib as a third-line and later therapy (ClinicalTrial.gov identifier NCT02995187). In addition, the studies of apatinib plus chemotherapy vs chemotherapy alone as the firstline treatment (ClinicalTrial.gov identifier NCT03164694) and the second-line treatment (ClinicalTrial.gov identifier NCT03256721) of NSCLC are underway.

\section{Esophageal squamous cell carcinoma}

Esophageal cancer is a complex disease that needs multidisciplinary therapy. The use of endoscopic procedures has improved the diagnosis and treatment of esophageal cancer. However, because the procedures are costly and often infeasible, many patients are diagnosed past the optimum stage for therapy. ${ }^{56}$

In 2017, a study reported that apatinib was effective for advanced esophageal squamous cell carcinoma (ESCC). Among 62 patients administered a dose of $500 \mathrm{mg} /$ day, 15 patients had a PR and 31 patients achieved SD, representing a response rate of $24.2 \%$ and a disease control rate of $74.2 \%$. The median PFS and OS were 3.8 and 7.0 months, respectively. Compared with second-line chemotherapy, apatinib showed a better impact on advanced ESCC. ${ }^{21}$

A study of apatinib and irinotecan combination treatment in unresectable or metastatic ESCC is currently in progress (ClinicalTrial.gov identifier NCT03251417) with the purpose of exploring the effectiveness as a second-line treatment for ESCC.

To sum up, the efficacy of apatinib in different trials is shown in Table 2. 
Table 2 The efficacy of apatinib in different trials

\begin{tabular}{|c|c|c|c|c|c|}
\hline \multirow[t]{2}{*}{ Trial } & \multicolumn{2}{|l|}{ Placebo } & \multicolumn{3}{|l|}{ Apatinib } \\
\hline & $\begin{array}{l}\text { Median OS } \\
\text { (months) }\end{array}$ & $\begin{array}{l}\text { Median PFS } \\
\text { (months) }\end{array}$ & Regimen & $\begin{array}{l}\text { Median OS } \\
\text { (months) }\end{array}$ & $\begin{array}{l}\text { Median PFS } \\
\text { (months) }\end{array}$ \\
\hline \multicolumn{6}{|l|}{ Gastric cancer } \\
\hline \multirow[t]{2}{*}{ Phase II0 } & 2.50 & 1.40 & $850 \mathrm{mg} / \mathrm{day}$ & 4.83 & 3.67 \\
\hline & & & $425 \mathrm{mg} / \mathrm{bid}$ & 4.27 & 3.2 \\
\hline Phase IIIII & 4.7 & 1.8 & $850 \mathrm{mg} /$ day & 6.5 & 2.6 \\
\hline \multicolumn{6}{|l|}{ Breast cancer } \\
\hline $\mathrm{TNBC}^{19}$ & & & $500 \mathrm{mg} / \mathrm{day}$ & 10.6 & 3.3 \\
\hline $\mathrm{MBC}^{45}$ & & & $500 \mathrm{mg} / \mathrm{day}$ & 10.3 & 4.9 \\
\hline \multicolumn{6}{|l|}{ Lung cancer } \\
\hline NSCLC 20 & & & $500 \mathrm{mg} /$ day & 6.0 & 4.2 \\
\hline EGFR wild type ${ }^{52}$ & & & $250-500 \mathrm{mg} /$ day & & 4.4 \\
\hline Esophageal squamous & & & $500 \mathrm{mg} /$ day & 7.0 & 3.8 \\
\hline cell carcinoma ${ }^{21}$ & & & & & \\
\hline
\end{tabular}

Abbreviations: bid, twice daily; EGFR, epidermal growth factor receptor; MBC, metastatic breast cancer; NSCLC, non-small-cell lung cancer; OS, overall survival; PFS, progression-free survival; TNBC, triple-negative breast cancer.

\section{The preclinical study of apatinib for other tumors}

Due to antiangiogenesis effects, apatinib could be applied for the treatment of various solid tumors. Currently, there are numerous preclinical and clinical studies looking at the efficacy and safety of apatinib.

In vitro, colon cancer cells (HCT116 and SW480) were treated with different concentrations of apatinib $(0,10,20$, $30,40$, and $50 \mu \mathrm{M})$. The investigator found that apatinib could not only inhibit proliferation, migration, and invasion of colon cancer cells, but also regulate the autophagy and apoptosis of the cells. ${ }^{28}$

In vivo, a murine model of nasopharyngeal carcinoma (NPC) was designed. In total, 132 nude mice, xenografted with CNE-2 NPC cells, were treated with six therapy regimens, including receiving apatinib alone and coadministration of apatinib and cisplatin (DDP), and so on. The results showed that concurrent administration of cisplatin and apatinib could treat NPC. ${ }^{57}$ Therefore, patients with metastatic NPC are presently being recruited in order to assess apatinib as a maintenance therapy for NPC (ClinicalTrial.gov identifier NCT03180476).

A series of trials were designed to determine the potency of apatinib for osteosarcoma. The growth of osteosarcoma cells could be suppressed because apatinib not only leads to cycle arrest, but also causes apoptosis and autophagy of the cells. In addition, immunoprecipitation verified that VEGFR-2 is directly bound with the signal transducer and activator of transcription 3 (STAT-3). Apatinib inhibits VEGFR-2 and subsequently downregulates STAT- 3 and BCL-2 in KHOS cells. The resulting low levels of VEGFR-2/STAT-3/BCL-2 led to apoptosis and autophagy of KHOS cells. ${ }^{27}$ Therefore, a Phase II/III study of apatinib for advanced osteosarcoma after failure of standard multimodal therapy is underway (ClinicalTrial.gov identifier NCT02711007).

A study of apatinib for the treatment of intrahepatic cholangiocarcinoma indicated that VEGF signaling (through $\mathrm{PI} 3 \mathrm{~K} / \mathrm{Akt} / \mathrm{mTOR}$ ) plays a role in the growth of the tumor. Apoptosis of tumor cells could be induced by blocking VEGFR-2. ${ }^{58}$ Therefore, a Phase III study of second-line treatment with apatinib in patients with intrahepatic cholangiocarcinoma is presently ongoing (ClinicalTrial.gov identifier NCT03251443).

The details of the ongoing clinical trials of apatinib are shown in Table 3. Furthermore, there are many other trials not mentioned in this article because of space limitations. Based on the posted study results, apatinib demonstrates positive results that make it a good prospect for treatment of various cancer.

\section{Adverse reactions to apatinib}

In contrast to placebo, the primary adverse reactions (ARs) of apatinib for GC are listed in Table 4. Hand-foot skin reaction, hypertension, and proteinuria were the most frequent ARs of antiangiogenic drugs. However, all of the ARs could be managed by reducing the dose of apatinib. ${ }^{18}$ As for other solid tumors, the ARs during treatment were analogous. ${ }^{21,54,59}$ In comparison to ramucirumab, apatinib showed a high rate of hypertension and proteinuria, but the occurrence rate of grade 3-4 events was low. ${ }^{18,60}$ In view of the present reports, apatinib shows acceptable safety.

According to another study, the patients enrolled with GC always had gastric surgery, so they appeared to have lower absorption of and exposure to apatinib. However, no formal 
Table 3 Ongoing clinical trials with apatinib

\begin{tabular}{|c|c|c|c|c|c|}
\hline Conditions & Identifier & Phase & Design & Agents & State \\
\hline \multirow[t]{6}{*}{ Gastric cancer } & NCT03333967 & - & Real-world study & Apatinib & Recruiting \\
\hline & NCT0327I073 & II & Open label: single group assignment & Apatinib and S-I & Recruiting \\
\hline & NCT03229096 & II & Open label: single group assignment & Apatinib and XELOX & Recruiting \\
\hline & NCT03। 44843 & II & Double blind, multicenter & Apatinib and paclitaxol & Recruiting \\
\hline & & & Randomized parallel assignment & & \\
\hline & NCT03007446 & II & Open label: single group assignment & Apatinib, oxaliplatin and S-I & Recruiting \\
\hline \multirow[t]{3}{*}{ Breast cancer } & NCT03086785 & II & $\begin{array}{l}\text { Open label: multicenter, observational } \\
\text { study }\end{array}$ & Apatinib and capecitabine & Recruiting \\
\hline & NCT03348098 & II & Open label: single group assignment & Apatinib and paclitaxel & Recruiting \\
\hline & NCT027684I5 & II & Open label: single group assignment & Apatinib and oral vinorelbine & Recruiting \\
\hline \multirow[t]{3}{*}{ Lung cancer } & NCT02995I87 & II & Open label: single group assignment & Apatinib & Recruiting \\
\hline & NCT03I64694 & II & $\begin{array}{l}\text { Open label: randomized parallel } \\
\text { assignment }\end{array}$ & $\begin{array}{l}\text { Apatinib, pemetrexed, and } \\
\text { carboplatin }\end{array}$ & Recruiting \\
\hline & NCT0325672I & II & $\begin{array}{l}\text { Double-blind randomized } \\
\text { parallel assignment }\end{array}$ & $\begin{array}{l}\text { Apatinib, docetaxel, and } \\
\text { pemetrexed }\end{array}$ & Recruiting \\
\hline $\begin{array}{l}\text { Esophageal squamous cell } \\
\text { carcinoma }\end{array}$ & NCT0325I4I7 & II & Open label: single group assignment & Apatinib and irinotecan & Recruiting \\
\hline Nasopharyngeal carcinoma & NCT03।80476 & II & Open label: single group assignment & Apatinib & Recruiting \\
\hline Osteosarcoma & NCT027II 007 & II/III & Open label: single group assignment & Apatinib & Recruiting \\
\hline Intrahepatic cholangiocarcinoma & NCT0325I443 & III & Open label: single group assignment & Apatinib & Recruiting \\
\hline
\end{tabular}

report explained the specific reason for the lower plasma concentration. However, it is known that the pharmacokinetics of apatinib in GC differs from that in other solid tumors. As a result, the dose of apatinib should be adjusted to maximize the benefits for patients with different solid tumors. ${ }^{36}$

Additionally, there are no reliable biomarkers to assess the response of apatinib in GC. A recent study analyzed the relevance between the early appearance of primary ARs and the clinical results in metastatic GC patients. The results revealed that patients who experienced ARs in the first 4 weeks achieved prolonged median OS and PFS. ${ }^{61}$ Therefore, the appearance of hypertension, proteinuria, and hand-foot syndrome in the first period of therapy could also be a feasible biomarker for the effectiveness of treatment in metastatic GC patients.

\section{Resistance of antiangiogenic drugs including apatinib}

Antiangiogenic drugs have achieved positive effects in clinical trials; however, drug resistance (DR) remains a problem that researchers cannot avoid. DR mainly includes the following mechanisms. ${ }^{62}$

First, infiltration of tumors by different stromal cells (including immature myeloid cells, endothelial progenitor cells, or fibroblasts) can mediate resistance to VEGF-targeted drugs. ${ }^{63-65}$ Second, angiogenesis is regulated by multiple

Table 4 The safety of apatinib for GC

\begin{tabular}{|c|c|c|c|c|c|c|c|c|}
\hline \multirow[t]{2}{*}{ Adverse effect } & \multicolumn{4}{|c|}{ Apatinib $(n=176)$} & \multicolumn{4}{|c|}{ Placebo $(n=91)$} \\
\hline & Total & $\%$ & $\begin{array}{l}\text { Grade } 3 \\
\text { or } 4\end{array}$ & $\%$ & Total & $\%$ & $\begin{array}{l}\text { Grade } 3 \\
\text { or } 4\end{array}$ & $\%$ \\
\hline Proteinuria & 84 & 47.7 & 4 & 2.3 & 15 & 16.5 & 0 & 0 \\
\hline Leukopenia & 71 & 40.3 & 3 & $\mathrm{I} .7$ & 8 & 8.8 & 0 & 0 \\
\hline Neutropenia & 66 & 37.5 & 10 & 5.7 & 9 & 9.9 & I & I.I \\
\hline Hypertension & 62 & 35.2 & 8 & 4.5 & 5 & 5.5 & 0 & 0 \\
\hline Hand-foot syndrome & 49 & 27.8 & 15 & 8.5 & 2 & 2.2 & 0 & 0 \\
\hline Elevated transaminase & 49 & 27.8 & 14 & 8.0 & 20 & 22.0 & 4 & 4.4 \\
\hline Anemia & 44 & 25.0 & $\mathrm{II}$ & 6.3 & 22 & 24.2 & 4 & 4.4 \\
\hline Thrombocytopenia & 44 & 25.0 & 5 & 2.8 & 6 & 6.6 & $\mathrm{I}$ & I.I \\
\hline Hyperbilirubinemia & 43 & 24.4 & 13 & 7.4 & 13 & 14.3 & 6 & 6.6 \\
\hline Fatigue & 36 & 20.5 & 5 & 2.8 & 13 & 14.3 & 2 & 2.2 \\
\hline Bleeding & 35 & 19.9 & 6 & 3.4 & 22 & 24.2 & 7 & 7.7 \\
\hline ALP increased & 35 & 19.9 & 5 & 2.8 & 14 & 15.4 & 0 & 0 \\
\hline
\end{tabular}

Note: Data from Li et al. ${ }^{18}$

Abbreviation: ALP, alkaline phosphatase. 
pathways. Although the VEGF pathway is the most important pathway, the compensatory effects of other pathways (such as c-kit and c-src) will gradually emerge as the treatment progresses. ${ }^{66,67}$ Third, tumors invade surrounding host tissues and form blood vessel-like structures and connect to host blood vessels, resulting in treatment failure. ${ }^{68,69}$ Fourth, the VEGF signaling system is complex and consists of five related ligands and three receptors. Because agents only work on one target, the efficacy will gradually decrease as treatment continues. ${ }^{5,6,70,71}$ Currently, there are no reports about DR to apatinib, but we can improve the treatment according to the common characteristics of antiangiogenic drugs. First, we can perform preconditioning to determine whether the tumor responds to VEGF-targeted therapy. Second, we can try to combine multiple antiangiogenic drugs to comprehensively suppress tumor progression. Of course, specific DR mechanisms still need to be explored. How to solve DR is one of the experimental directions in the future.

\section{Discussion}

Since precision medicine has become the hotspot of cancer therapy, multifarious molecular targets turn into crucial objects of study and the discovery of a specific target can be a breakthrough in antitumor research. Detection of VEGF signaling promoted the comprehension of the mechanism of angiogenesis. ${ }^{5,70,72}$ Today, the agents that target VEGFR are being increasingly developed and put into use. Compared to drugs that target VEGFR-1, the antiangiogenesis ability of apatinib, which targets VEGFR-2, is affirmative. ${ }^{17,25,73}$ Apatinib has been experimentally used in many cancer types and has proven to be a promising treatment in preclinical and clinical trials. For the moment, it has been shown that the curative effect of apatinib is slightly better than that of other antiangiogenic drugs such as ramucirumab, bevacizumab, and sunitinib. ${ }^{39}$ In addition, apatinib can reverse the MDR caused by EGFR-TKIs and ABC proteins, ${ }^{34}$ and it has a positive safety profile. Therefore, apatinib can be a promising treatment for different advanced cancers. Currently, extensive Phase II studies regarding different tumors are being conducted, and they will provide more detailed and accurate supporting data on the clinical application of apatinib.

However, there are a few issues that still require clarification.

First, the specific pathological make-up of the participants and the previous treatment plan are inexplicit. ${ }^{18}$ In fact, the effect of treatment will be influenced by these two factors. It is necessary to make it clear which type of pathological patient is more sensitive to treatment and whether the previous treatment plan has influence on subsequent medications. These detailed data are helpful to guide future patient selection, so they should be collected in subsequent trials.

Second, there are no clear biomarkers used to predict patient response to treatment and to determine prognosis. Every mature treatment program has corresponding biomarkers to evaluate the current status of patients. This is very important for the adjustment of individualized treatment plans. Therefore, researchers need to carefully analyze the changes in each indicator during treatment and identify the characteristic indicators. As mentioned earlier, early ARs may be a potential biomarker. ${ }^{61}$ In addition, biomarkers for other antiangiogenic drugs have been proposed, such as circulating markers and single-nucleotide polymorphisms, ${ }^{62}$ but whether those biomarkers specifically relate to apatinib needs requires further exploration.

Third, patients' quality of life should be taken seriously. In current times, patients and their families have an urgent need to improve quality of life. Doctors can no longer use therapeutic effects as an evaluation indicator for treatment; they also need to be responsible for patient mental health, social skills, and quality of life. How to achieve the best therapeutic effect while minimizing adverse drug reactions is the goal of further research. In addition to hematologic concerns, cardiac toxicity is also a common serious AR of antiangiogenic drugs. ${ }^{74,75}$ Although the incidence of cardiac toxicity is low in apatinib treatment, ${ }^{18}$ the mechanism in this area needs further elucidation in order to properly solve the problem.

Fourth, the cost-effectiveness for the patient needs to be considered. A study developed a Markov model to assess the incremental cost-effectiveness ratio of apatinib to placebo. The study found that apatinib was currently not a cost-effective option for GC patients in China in whom second-line chemotherapy had failed. ${ }^{76}$ However, the efficacy of apatinib was not inferior to that of ramucirumab (another VEGFR-TKI approved by the US Food and Drug Administration for advanced GC), and apatinib was an oral agent, which would also reduce costs. Therefore, apatinib is still a good option due to its positive clinical value. In addition, a charitable assistance program, the China Pharmaceutical Innovation and Research Development Association (PhIRDA) is underway, which greatly reduces the financial burden on patients. ${ }^{76}$ The improvement of incremental costeffectiveness ratio can be achieved through value-based pricing or increasing the effect of the therapeutic regimen. Therefore, new assistance programs or medical insurance are expected. Furthermore, multidisciplinary treatment is a promising means in oncotherapy. More research is required to explore the optimal therapy, such as the combination of 
apatinib and other antitumor drugs or therapy methods. We look forward to finding an effective way to maximize the benefit to patients.

\section{Conclusion}

Apatinib is now acknowledged as a novel oral small-molecule TKI in China. Due to antiangiogenesis activity, it could be applied to a variety of solid tumors. Currently, several studies are in progress, and many of them suggest that apatinib has good efficacy and safety in clinical trials. The ARs are tolerated, compared with other target agents, and the majority of them can be managed. Therefore, apatinib is a viable choice for the treatment of solid tumors.

\section{Acknowledgments}

This work was supported by the Taishan Scholar foundation (No tshw201502061), the Qingdao People's Livelihood Science and Technology Program (16-6-2-3-nsh), and the third batch of the Qingdao Entrepreneurial Innovation Leading Talent Program.

\section{Disclosure}

The authors report no conflicts of interest in this work.

\section{References}

1. Chen W, Zheng R, Baade PD, et al. Cancer statistics in China, 2015. CA Cancer J Clin. 2016;66(2):115-132.

2. van Hagen P, Hulshof MC, van Lanschot JJ, et al. Preoperative chemoradiotherapy for esophageal or junctional cancer. $N$ Engl J Med. 2012;366(22):2074-2084.

3. Le Tourneau C, Delord JP, Gonçalves A, et al. Molecularly targeted therapy based on tumour molecular profiling versus conventional therapy for advanced cancer (SHIVA): a multicentre, open-label, proofof-concept, randomised, controlled phase 2 trial. Lancet Oncol. 2015; 16(13):1324-1334.

4. Gotwals P, Cameron S, Cipolletta D, et al. Prospects for combining targeted and conventional cancer therapy with immunotherapy. Nat Rev Cancer. 2017;17(5):286-301.

5. Carmeliet $P$, Jain RK. Angiogenesis in cancer and other diseases. Nature. 2000;407(6801):249-257.

6. Roudsari LC, West JL. Studying the influence of angiogenesis in in vitro cancer model systems. Adv Drug Deliv Rev. 2016;97:250-259.

7. Ferrara N, Gerber HP, Lecouter J. The biology of VEGF and its receptors. Nat Med. 2003;9(6):669-676.

8. Ellis LM, Hicklin DJ. VEGF-targeted therapy: mechanisms of antitumour activity. Nat Rev Cancer. 2008;8(8):579-591.

9. Shibuya M. Vascular endothelial growth factor and its receptor system: physiological functions in angiogenesis and pathological roles in various diseases. J Biochem. 2013;153(1):13-19.

10. Goel HL, Mercurio AM. VEGF targets the tumour cell. Nat Rev Cancer. 2013;13(12):871-882.

11. Ebos JM, Bocci G, Man S, et al. A naturally occurring soluble form of vascular endothelial growth factor receptor 2 detected in mouse and human plasma. Mol Cancer Res. 2004;2(6):315-326.

12. Holmes K, Roberts OL, Thomas AM, Cross MJ. Vascular endothelial growth factor receptor-2: structure, function, intracellular signalling and therapeutic inhibition. Cell Signal. 2007;19(10):2003-2012.
13. Geng R, Li J. Apatinib for the treatment of gastric cancer. Expert Opin Pharmacother. 2015;16(1):117-122.

14. Brower V. Apatinib in treatment of refractory gastric cancer. Lancet Oncol. 2016;17(4):e137.

15. Ding L, Li QJ, You KY, Jiang ZM, Yao HR. The Use of Apatinib in Treating Nonsmall-Cell Lung Cancer: Case Report and Review of Literature. Medicine. 2016;95(20):e3598.

16. Li F, Liao Z, Zhao J, et al. Efficacy and safety of Apatinib in stage IV sarcomas: experience of a major sarcoma center in China. Oncotarget. 2017;8(38):64471-64480.

17. Scott AJ, Messersmith WA, Jimeno A. Apatinib: a promising oral antiangiogenic agent in the treatment of multiple solid tumors. Drugs Today. 2015;51(4):223-229.

18. Li J, Qin S, Xu J, et al. Randomized, Double-Blind, Placebo-Controlled Phase III Trial of Apatinib in Patients With Chemotherapy-Refractory Advanced or Metastatic Adenocarcinoma of the Stomach or Gastroesophageal Junction. J Clin Oncol. 2016;34(13):1448-1454.

19. Hu X, Zhang J, Xu B, et al. Multicenter phase II study of apatinib, a novel VEGFR inhibitor in heavily pretreated patients with metastatic triple-negative breast cancer. Int J Cancer. 2014;135(8):1961-1969.

20. Song Z, Yu X, Lou G, Shi X, Zhang Y. Salvage treatment with apatinib for advanced non-small-cell lung cancer. Onco Targets Ther. 2017;10: $1821-1825$.

21. Li J, Wang L. Efficacy and safety of apatinib treatment for advanced esophageal squamous cell carcinoma. Onco Targets Ther. 2017;10: 3965-3969.

22. Bergers G, Brekken R, Mcmahon G, et al. Matrix metalloproteinase-9 triggers the angiogenic switch during carcinogenesis. Nat Cell Biol. 2000;2(10):737-744.

23. Plate KH, Breier G, Millauer B, Ullrich A, Risau W. Up-regulation of vascular endothelial growth factor and its cognate receptors in a rat glioma model of tumor angiogenesis. Cancer Res. 1993;53(23): $5822-5827$

24. Roviello G, Ravelli A, Polom K, et al. Apatinib: A novel receptor tyrosine kinase inhibitor for the treatment of gastric cancer. Cancer Lett. 2016;372(2):187-191.

25. Tian S, Quan H, Xie C, et al. YN968D1 is a novel and selective inhibitor of vascular endothelial growth factor receptor-2 tyrosine kinase with potent activity in vitro and in vivo. Cancer Sci. 2011;102(7): 1374-1380.

26. Li X, Xu A, Li H, Zhang B, Cao B, Huang J. Novel role of apatinib as a multi-target RTK inhibitor in the direct suppression of hepatocellular carcinoma cells. Biochim Biophys Acta. 2018;1864(5 Pt A):1693-1701.

27. Liu K, Ren T, Huang Y, et al. Apatinib promotes autophagy and apoptosis through VEGFR2/STAT3/BCL-2 signaling in osteosarcoma. Cell Death Dis. 2017;8(8):e3015.

28. Lu W, Ke H, Qianshan D, Zhen W, Guoan X, Honggang Y. Apatinib has anti-tumor effects and induces autophagy in colon cancer cells. Iran J Basic Med Sci. 2017;20(9):990-995.

29. Peng H, Zhang Q, Li J, et al. Apatinib inhibits VEGF signaling and promotes apoptosis in intrahepatic cholangiocarcinoma. Oncotarget. 2016;7(13):17220-17229.

30. Yin L, Wang J, Huang FC, et al. Inhibitory effect of apatinib on HCT-116 cells and its mechanism. Nan Fang Yi Ke Da Xue Xue Bao. 2017;37(3):367-372.

31. Zhang H, Cao Y, Chen Y, Li G, Yu H. Apatinib promotes apoptosis of the SMMC-7721 hepatocellular carcinoma cell line via the PI3K/Akt pathway. Oncol Lett. 2018;15(4):5739-5743.

32. Ozben T. Mechanisms and strategies to overcome multiple drug resistance in cancer. FEBS Lett. 2006;580(12):2903-2909.

33. Pérez-Tomás R. Multidrug resistance: retrospect and prospects in anticancer drug treatment. Curr Med Chem. 2006;13(16):1859-1876.

34. Mi YJ, Liang YJ, Huang HB, et al. Apatinib (YN968D1) reverses multidrug resistance by inhibiting the efflux function of multiple ATPbinding cassette transporters. Cancer Res. 2010;70(20):7981-7991.

35. Ding J, Chen X, Gao Z, et al. Metabolism and pharmacokinetics of novel selective vascular endothelial growth factor receptor-2 inhibitor apatinib in humans. Drug Metab Dispos. 2013;41(6):1195-1210. 
36. Yu M, Gao Z, Dai X, et al. Population Pharmacokinetic and Covariate Analysis of Apatinib, an Oral Tyrosine Kinase Inhibitor, in Healthy Volunteers and Patients with Solid Tumors. Clin Pharmacokinet. 2017;56(1):65-76.

37. Liu X, Zhang Y, Chen Q, et al. Pharmacokinetic Drug Interactions of Apatinib With Rifampin and Itraconazole. J Clin Pharmacol. 2017.

38. Zong L, Abe M, Seto Y, Ji J. The challenge of screening for early gastric cancer in China. Lancet. 2016;388(10060):2606.

39. Aoyama T, Yoshikawa T. Targeted therapy: Apatinib - new third-line option for refractory gastric or GEJ cancer. Nat Rev Clin Oncol. 2016; 13(5):268-270.

40. Li J, Zhao X, Chen L, et al. Safety and pharmacokinetics of novel selective vascular endothelial growth factor receptor-2 inhibitor YN968D1 in patients with advanced malignancies. BMC Cancer. 2010; 10:529.

41. Li J, Qin S, Xu J, et al. Apatinib for chemotherapy-refractory advanced metastatic gastric cancer: results from a randomized, placebo-controlled, parallel-arm, phase II trial. J Clin Oncol. 2013;31(26):3219-3225.

42. Huang L, Wei Y, Shen S, et al. Therapeutic effect of apatinib on overall survival is mediated by prolonged progression-free survival in advanced gastric cancer patients. Oncotarget. 2017;8(17):29346-29354.

43. Zhao TT, Xu H, Xu HM, Hm X, et al. The efficacy and safety of targeted therapy with or without chemotherapy in advanced gastric cancer treatment: a network meta-analysis of well-designed randomized controlled trials. Gastric Cancer. 2018;21(3):361-371.

44. Bianchini G, Balko JM, Mayer IA, Sanders ME, Gianni L. Triplenegative breast cancer: challenges and opportunities of a heterogeneous disease. Nat Rev Clin Oncol. 2016;13(11):674-690.

45. Lin $\mathrm{Y}, \mathrm{Wu} \mathrm{Z}$, Zhang J, et al. Apatinib for metastatic breast cancer in non-clinical trial setting: Satisfying efficacy regardless of previous antiangiogenic treatment. Tumour Biol. 2017;39(6):1010428317711033.

46. Ebos JM, Lee CR, Cruz-Munoz W, Bjarnason GA, Christensen JG, Kerbel RS. Accelerated metastasis after short-term treatment with a potent inhibitor of tumor angiogenesis. Cancer Cell. 2009;15(3): 232-239.

47. Chung AS, Kowanetz M, Wu X, et al. Differential drug class-specific metastatic effects following treatment with a panel of angiogenesis inhibitors. J Pathol. 2012;227(4):404-416.

48. Welti JC, Powles T, Foo S, et al. Contrasting effects of sunitinib within in vivo models of metastasis. Angiogenesis. 2012;15(4):623-641.

49. Cooke VG, Lebleu VS, Keskin D, et al. Pericyte depletion results in hypoxia-associated epithelial-to-mesenchymal transition and metastasis mediated by met signaling pathway. Cancer Cell. 2012;21(1): 66-81.

50. Rotow J, Bivona TG. Understanding and targeting resistance mechanisms in NSCLC. Nat Rev Cancer. 2017;17(11):637-658.

51. Janku F, Garrido-Laguna I, Petruzelka LB, Stewart DJ, Kurzrock R. Novel therapeutic targets in non-small cell lung cancer. J Thorac Oncol. 2011;6(9):1601-1612.

52. Zeng DX, Wang CG, Lei W, Huang JA, Jiang JH. Efficiency of low dosage apatinib in post-first-line treatment of advanced lung adenocarcinoma. Oncotarget. 2017;8(39):66248-66253.

53. Fang SC, Zhang HT, Zhang YM, Xie WP. Apatinib as post second-line therapy in EGFR wild-type and ALK-negative advanced lung adenocarcinoma. Onco Targets Ther. 2017;10:447-452.

54. Li F, Zhu T, Cao B, Wang J, Liang L. Apatinib enhances antitumour activity of EGFR-TKIs in non-small cell lung cancer with EGFR-TKI resistance. Eur J Cancer. 2017;84:184-192.

55. Peng Y, Cui H, Liu Z, et al. Apatinib to combat EGFR-TKI resistance in an advanced non-small cell lung cancer patient with unknown EGFR status: a case report. Onco Targets Ther. 2017;10:2289-2295.

56. Lagergren J, Smyth E, Cunningham D, Lagergren P. Oesophageal cancer. Lancet. 2017;390(10110):2383-2396.

57. Peng QX, Han YW, Zhang YL, et al. Apatinib inhibits VEGFR-2 and angiogenesis in an in vivo murine model of nasopharyngeal carcinoma. Oncotarget. 2017;8(32):52813-52822.
58. Peng $\mathrm{H}$, Zhang Q, Li J, Jl L, et al. Apatinib inhibits VEGF signaling and promotes apoptosis in intrahepatic cholangiocarcinoma. Oncotarget. 2016;7(13):17220-17229.

59. Hu X, Cao J, Hu W, et al. Multicenter phase II study of apatinib in nontriple-negative metastatic breast cancer. BMC Cancer. 2014;14:820.

60. Fuchs CS, Tomasek J, Yong CJ, et al. Ramucirumab monotherapy for previously treated advanced gastric or gastro-oesophageal junction adenocarcinoma (REGARD): an international, randomised, multicentre, placebo-controlled, phase 3 trial. Lancet. 2014;383(9911):31-39.

61. Liu X, Qin S, Wang Z, et al. Early presence of anti-angiogenesisrelated adverse events as a potential biomarker of antitumor efficacy in metastatic gastric cancer patients treated with apatinib: a cohort study. J Hematol Oncol. 2017;10(1):153.

62. Vasudev NS, Reynolds AR. Anti-angiogenic therapy for cancer: current progress, unresolved questions and future directions. Angiogenesis. 2014;17(3):471-494.

63. Shojaei F, Wu X, Malik AK, et al. Tumor refractoriness to anti-VEGF treatment is mediated by $\mathrm{CD} 11 \mathrm{~b}+\mathrm{Gr} 1+$ myeloid cells. Nat Biotechnol. 2007;25(8):911-920.

64. Chung AS, Wu X, Zhuang G, et al. An interleukin-17-mediated paracrine network promotes tumor resistance to anti-angiogenic therapy. Nat Med. 2013;19(9):1114-1123.

65. Shaked Y, Ciarrocchi A, Franco M, et al. Therapy-Induced Acute Recruitment of Circulating Endothelial Progenitor Cells to Tumors. Science. 2006;313(5794):1785-1787.

66. Lieu C, Heymach J, Overman M, Tran H, Kopetz S. Beyond VEGF: inhibition of the fibroblast growth factor pathway and antiangiogenesis. Clin Cancer Res. 2011;17(19):6130-6139.

67. Li JL, Sainson RC, Oon CE, et al. DLL4-Notch signaling mediates tumor resistance to anti-VEGF therapy in vivo. Cancer Res. 2011;71(18): 6073-6083.

68. Kilarski WW, Samolov B, Petersson L, Kvanta A, Gerwins P. Biomechanical regulation of blood vessel growth during tissue vascularization. Nat Med. 2009;15(6):657-664.

69. Du R, Lu KV, Petritsch C, et al. HIF1alpha induces the recruitment of bone marrow-derived vascular modulatory cells to regulate tumor angiogenesis and invasion. Cancer Cell. 2008;13(3):206-220.

70. Goel HL, Mercurio AM. VEGF targets the tumour cell. Nat Rev Cancer. 2013;13(12):871-882.

71. Tugues S, Koch S, Gualandi L, Li X, Claesson-Welsh L. Vascular endothelial growth factors and receptors: anti-angiogenic therapy in the treatment of cancer. Mol Aspects Med. 2011;32(2):88-111.

72. Hicklin DJ, Ellis LM. Role of the vascular endothelial growth factor pathway in tumor growth and angiogenesis. J Clin Oncol. 2005;23(5):1011-1027.

73. Zhang H. Apatinib for molecular targeted therapy in tumor. Drug Des Devel Ther. 2015;9:6075-6081.

74. Telli ML, Witteles RM, Fisher GA, Srinivas S. Cardiotoxicity associated with the cancer therapeutic agent sunitinib malate. Ann Oncol. 2008;19(9):1613-1618.

75. Chen XL, Lei YH, Liu CF, et al. Angiogenesis inhibitor bevacizumab increases the risk of ischemic heart disease associated with chemotherapy: a meta-analysis. PLoS One. 2013;8(6):e66721.

76. Chen HD, Zhou J, Wen F, et al. Cost-effectiveness analysis of apatinib treatment for chemotherapy-refractory advanced gastric cancer. J Cancer Res Clin Oncol. 2017;143(2):361-368.

77. Bang YJ, Kang YK, Kang WK, et al. Phase II study of sunitinib as second-line treatment for advanced gastric cancer. Invest New Drugs. 2011;29(6):1449-1458.

78. Pavlakis N, Sjoquist KM, Martin AJ, et al. Regorafenib for the Treatment of Advanced Gastric Cancer (INTEGRATE): A Multinational PlaceboControlled Phase II Trial. J Clin Oncol. 2016;34(23):2728-2735.

79. Martin-Richard M, Gallego R, Pericay C, et al. Multicenter phase II study of oxaliplatin and sorafenib in advanced gastric adenocarcinoma after failure of cisplatin and fluoropyrimidine treatment. A GEMCAD study. Invest New Drugs. 2013;31(6):1573-1579. 
80. Ohtsu A, Shah MA, van Cutsem E, et al. Bevacizumab in combination with chemotherapy as first-line therapy in advanced gastric cancer: a randomized, double-blind, placebo-controlled phase III study. J Clin Oncol. 2011;29(30):3968-3976.

81. Wilke H, Muro K, van Cutsem E, et al. Ramucirumab plus paclitaxel versus placebo plus paclitaxel in patients with previously treated advanced gastric or gastro-oesophageal junction adenocarcinoma (RAINBOW): a double-blind, randomised phase 3 trial. Lancet Oncol. 2014;15(11):1224-1235.
82. Yoon HH, Bendell JC, Braiteh FS, et al. Ramucirumab combined with FOLFOX as front-line therapy for advanced esophageal, gastroesophageal junction, or gastric adenocarcinoma: a randomized, double-blind, multicenter Phase II trial. Ann Oncol. 2016;27(12): 2196-2203.

\section{Publish your work in this journal}

OncoTargets and Therapy is an international, peer-reviewed, open access journal focusing on the pathological basis of all cancers, potential targets for therapy and treatment protocols employed to improve the management of cancer patients. The journal also focuses on the impact of management programs and new therapeutic agents and protocols on

\section{Dovepress}

patient perspectives such as quality of life, adherence and satisfaction. The manuscript management system is completely online and includes a very quick and fair peer-review system, which is all easy to use. Visit http://www.dovepress.com/testimonials.php to read real quotes from published authors.

Submit your manuscript here: http://www.dovepress.com/oncotargets-and-therapy-journal 